\title{
Distribution-Based Classification Method for Baseline Correction of Metabolomic 1D Proton Nuclear Magnetic Resonance Spectra
}

\author{
Kuo-Ching Wang, ${ }^{\dagger, \|, \perp, \#, \nabla}$ San-Yuan Wang, ${ }^{\ddagger}, \|$ Ching-hua Kuo, ${ }^{\S, \|}$ and Yufeng J. Tseng ${ }^{* \dagger, \dagger, \S, \|}$ \\ ${ }^{\dagger}$ Graduate Institute of Biomedical Electronics and Bioinformatics, National Taiwan University, No. 1 Sec. 4, Roosevelt Road, Taipei, \\ Taiwan 106 \\ ${ }^{\ddagger}$ Department of Computer Science and Information Engineering, National Taiwan University, Taipei, Taiwan \\ ${ }^{\S}$ Department of Pharmacy, National Taiwan University, Taipei, Taiwan \\ ${ }^{\|}$The Metabolomics Group, National Taiwan University, Taipei, Taiwan \\ ${ }^{\perp}$ School of Medicine, Fu Jen Catholic University, Taiwan \\ ${ }^{\#}$ School of Medicine, Taipei Medical University, Taipei, Taiwan \\ ${ }^{\nabla}$ Department of Anesthesia, Shin-Kong Wu Ho-Su Memorial Hospital, Taipei, Taiwan
}

Supporting Information

ABSTRACT: Baseline distortion in $1 \mathrm{D}{ }^{1} \mathrm{H}$ NMR data complicates the quantification of individual components of biofluids in metabolomic experiments. Current $1 \mathrm{D}{ }^{1} \mathrm{H}$ NMR baseline correction methods usually require manual parameter and filter tuning by experienced users to obtain desirable results from complex metabolomic spectra, thus becoming prone to correction variation and biased quantification. We

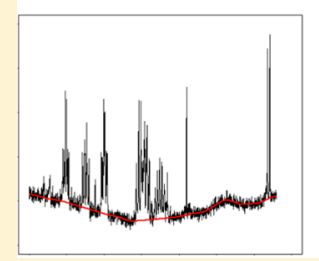

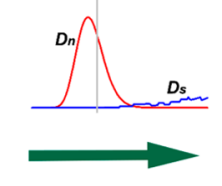

Distribution-based Baseline Correction

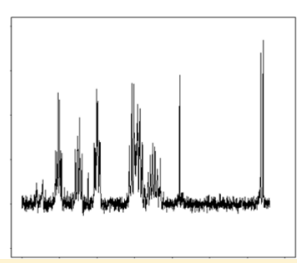
present a novel alternative method, BaselineCorrector, for automatically estimating the baselines of $1 \mathrm{D}{ }^{1} \mathrm{H}$ NMR metabolomic data. By collecting the standard deviations of spectral intensities, using a moving window to slide through a spectrum, BaselineCorrector can model the distribution of noise standard deviation as a derived chi-squared distribution in each window and then determine optimal parameters for least-error classification of signal and noise. Due to the universal property of noise distributions, BaselineCorrector can robustly recognize the baseline segments in various spectra. In addition to the commonly used 1D NOESY and CPMG pulse sequences, BaselineCorrector also provides an algorithm for correcting diffusion-edited NMR spectra. Using its classification model, BaselineCorrector is able to preserve low signal peaks and correctly handle wide, overlapping peaks in complex metabolomic spectra.

$\mathrm{M}$ etabolomic studies based on proton nuclear magnetic resonance $\left({ }^{1} \mathrm{H}\right.$ NMR) experiments are widely employed in biomedical research. ${ }^{1-10}$ However, metabolomic ${ }^{1} \mathrm{H}$ NMR spectra are frequently subject to baseline drifts arising from diverse sources, including instrumental instability, strong freeinduction decay, etc. ${ }^{11-15}$ The nonzero baseline intensities significantly disturb the measured signal intensities of interests in metabolomic ${ }^{1} \mathrm{H}$ NMR spectra because the acquired spectral intensities are presumed to be the sum of the true signal intensities, baseline intensities, and random noise. Therefore, the shifting baseline values should be cautiously corrected to zero to reduce errors in the subsequent chemometric analysis in metabolomics studies.

Most metabolomic NMR studies use frequency-domain baseline corrections to identify the true baseline segments (signal-free regions) and fit a smooth curve to these regions to estimate the baseline. ${ }^{16}$ These methods offset residual baseline distortion and improve quantification accuracy after careful adjustments of acquisition settings and time-domain parameters. ${ }^{16-27}$ Because the metabolomic NMR spectra are usually complicated by a wide variety of signals and baseline artifacts, identification of the signal-free regions is not easy. Distinguishing between signal and signal-free regions becomes a very critical step affecting quality of the baseline correction.

Parameter adjustments to identify signal-free regions by users are often required to produce desired results in current baseline correction methods. ${ }^{16-21,27,28}$ For example, Dietrich et al. ${ }^{17}$ smoothed spectra using an average filter and then calculated the first derivative of each spectrum. Subsequently, this firstderivative pseudo spectrum was converted to a pseudo power spectrum, to which an iterative threshold algorithm was applied to identify the signal-free regions. In Dietrich's method, users must determine a suitable width of the average filter for the best baseline correction. Cobas et al. ${ }^{18}$ used a continuous wavelet transform to combine the smoothing and derivative calculations into a single mathematical function for peak/baseline detections. The scale parameter used in the transformation must be tuned for spectra with different noise levels in this

Received: November 6, 2012

Accepted: December 18, 2012

Published: December 18, 2012 
method. Zhang et al. ${ }^{27}$ implemented a penalized least-squares method (airPLS) to iteratively reweight and eliminate the spectral points of the peaks and smooth the preserved baseline points of the spectrum to produce the baseline. In the airPLS algorithm, the user must choose the parameter value to balance the fidelity (retaining the exactness of the original spectrum) and smoothness during the iteration process. Golotvin et al. ${ }^{19}$ identified signal-free regions by calculating the difference between maximal and minimal intensities within a given segment and then used an algorithm to compare this difference with the standard deviation of noise multiplied by a threshold constant, the selection of which determined the quality/ accuracy of the method. Bao et al. ${ }^{28}$ combined three methods derived from the works of Cobas et al., Golotvin et al., and Dietrich et al. and a peak shape function to recognize the signalfree regions. An iterative weighted function was used to correct the negative signal regions and construct the baseline curves in Bao's work.

An alternative approach that does not rely on identification of signal-free regions via a penalized parametric smoothing model for baseline correction was proposed by Xi and Rocke. ${ }^{20}$ Their model implements a scoring function with three components: (1) the sum of baseline intensities, (2) a smoothness penalty, and (3) a negativity penalty. Maximizing this function produces the estimated baseline. Thus, parameters must also be optimized in $\mathrm{Xi}$ and Rocke's method to achieve the best baseline fit. ${ }^{26}$

In addition to the algorithms described above, several commercial NMR spectra processing softwares are capable of correcting baseline automatically, such as Chenomx (Chenomx Inc., Canada), ${ }^{21}$ ACD NMR Spectroscopy Softwares (Advanced Chemistry Development, Inc., ACD/Laboratories, Canada), MNova (Mestrelab Research SL, USA), iNMR, ${ }^{29}$ etc. To improve the automatic baseline correction, all of them offer interactive user interfaces for experienced users to manually select the baseline points or correction parameters to adjust the results.

However, parameters tuning for baseline correction or manual baseline readjustments are nearly inevitable due to the complex metabolomic NMR spectra from biofluids composed of overlapping resonances from thousands of metabolites. As a result, the signal and signal-free regions in these spectra are obscured and difficult to discriminate, causing real features to be commonly mistaken for distorted baseline artifacts. Thus, the use of default parameter values in the above algorithms or software packages frequently result in inadequate baseline corrections since they were mostly designed for pure compounds. There are currently no proposed rules for objective parameter selections in the baseline correction processes for complex metabolomic ${ }^{1} \mathrm{H}$ NMR spectra. In most current metabolomics studies, a better baseline suitable for subsequent biomarker identification and quantification relies heavily on the users' experience and preference. Therefore, the baseline correction outcomes generated by different people or laboratories may contain large correction variations and are possible to cause biases in quantification.

To reduce the uncertainties of typical baseline estimations that result from personal parameter selections, we modeled distribution of standard deviations calculated from each spectrum's noise to derive a novel baseline correction algorithm, BaselineCorrector, in the frequency-domain. Via a distribution model, BaselineCorrector can accurately estimate the percentage of noise points utilized for baseline building to improve the signal/noise classification. BaselineCorrector has the advantage of statistically optimized classification parameters without manual adjustment and can minimize the baseline errors frequently associated with the metabolomic spectra.

\section{THEORETICAL BASIS}

Our distribution-based baseline correction model, BaselineCorrector, consists of a main algorithm for general $1 \mathrm{D}{ }^{1} \mathrm{H}$ NMR spectra and an additional algorithm designed for diffusionedited spectra. ${ }^{30}$ Processing of the main algorithm includes the following: (1) calculating the standard deviations of the spectral points within a window that is sliding across a spectrum; (2) modeling the standard deviation distribution of noise; (3) classifying each sliding window as a signal window or a noise window according to the distribution model of (2); (4) classifying the spectral points in each window according to the window type; and (5) constructing the baseline. Processing of the additional algorithm for a diffusion-edited spectrum includes the following: (1) using the main algorithm to build a preliminary baseline; (2) calculating a baseline fluctuation threshold from the downfield spectrum (6-10 ppm); (3) reclassifying the spectral points of the upfield spectrum (0-6 ppm) according to the threshold; and (4) rebuilding and correcting the baseline with the new classification. The workflow of BaselineCorrector is shown in Scheme 1. The pseudo code of the BaselineCorrector algorithm and the detailed theory for diffusion-edited spectra are provided in the Supporting Information.

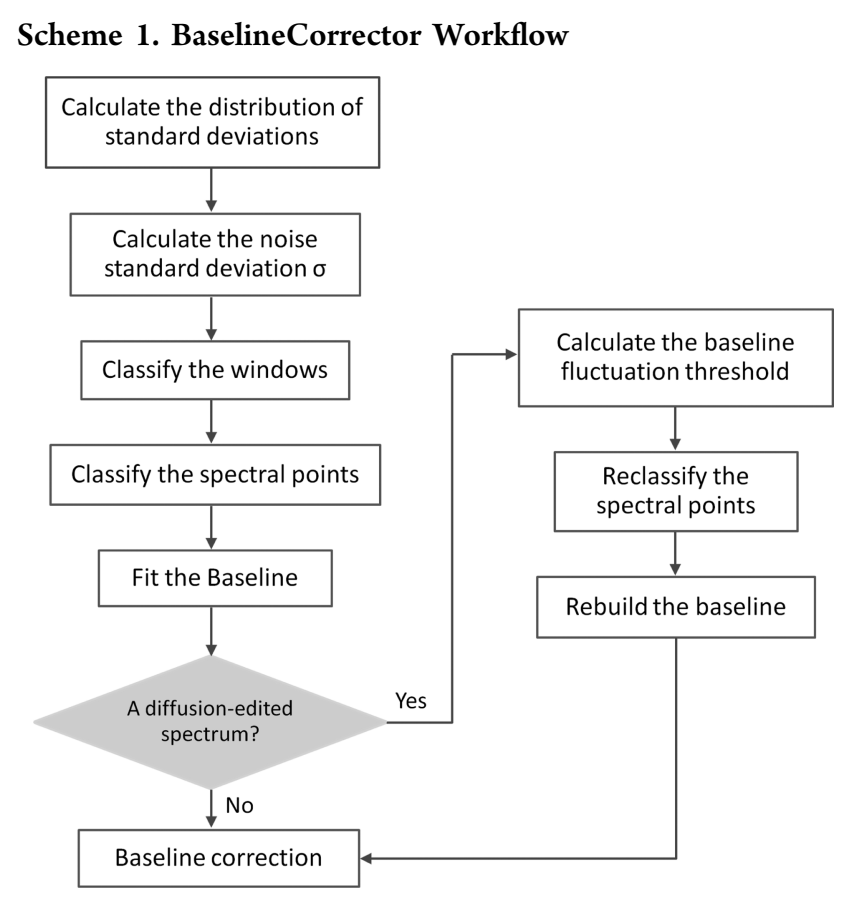

Distributions of Standard Deviation. In this study, BaselineCorrector turns the problems of baseline recognition into problems of statistical classification. Before the classification, we first assume that there are two types of points in a spectrum: "noise point" and "signal point". A "noise point" is defined as a spectral point that does not contain any signal intensity, and a "signal point" as a spectral point that contains a signal component. BaselineCorrector walks a fixed-length sliding window, point-by-point, across a spectrum and obtains 
a set of windows containing noise points, signal points, or both. On the basis of the above definition, we assume there are two kinds of windows obtained: $W_{\mathrm{n}}$ (a window containing purely noise points) and $W_{s}$ (a window containing at least one signal point). We denominate the values of standard deviation calculated from $W_{\mathrm{n}}$ as "noise standard deviation" and the values of standard deviation calculated from $W_{s}$ as "signal standard deviation".

Following the above definitions, there are three types of probability distributions, $D_{\mathrm{n}}, D_{\mathrm{s}}$, and $D_{\mathrm{m}}$, defined as follows: $D_{\mathrm{n}}$, the probability distribution of noise standard deviation; $D_{s}$, the probability distribution of signal standard deviation; $D_{\mathrm{m}}$, mixture distribution of $D_{\mathrm{n}}$ and $D_{s}$.

The mixture distribution, $D_{\mathrm{m}}$, is the real distribution which can be directly obtained from the standard deviation values of all sliding windows, while the distributions of $D_{\mathrm{n}}$ and $D_{\mathrm{s}}$ are conceptual distributions. Theoretically, $D_{\mathrm{m}}$ can be further decomposed into a combination of $D_{\mathrm{n}}$ and $D_{\mathrm{s}}$.

To model $D_{n}$, we define $X_{1}, \ldots, X_{n}$ as $n$ independent and identically distributed normal random variables. With $\mu$ defined as the expected value of $X$ and $n$ as the point number in the sliding window, we further define the random variables $V$ and $S$ as follows:

$$
\begin{aligned}
& V=\sum(X-\mu)^{2} / n, \quad \text { the variance of } X \\
& S=\left(\sum(X-\mu)^{2} / n\right)^{1 / 2}, \quad \text { the standard deviation of } X
\end{aligned}
$$

Because the distribution of random variable $X$ is a normal distribution, the distribution of $\Sigma(X-\mu)^{2}$ is a chi-squared distribution with $n$ degrees of freedom. The distribution of $V$ can be derived from the chi-squared distribution, and the distribution of $S$ can be derived from $V$. Because the distribution of noise in a spectrum can be accurately fitted as a normal distribution ${ }^{31}$ and because $D_{n}$ is the distribution of noise standard deviation, $D_{\mathrm{n}}$ can be modeled with $S$. The distributions of $S$ and the theoretical $D_{\mathrm{n}}$ from the statistical model are bell-shaped (Figure 1). In contrast to $W_{\mathrm{n}}$, signals are

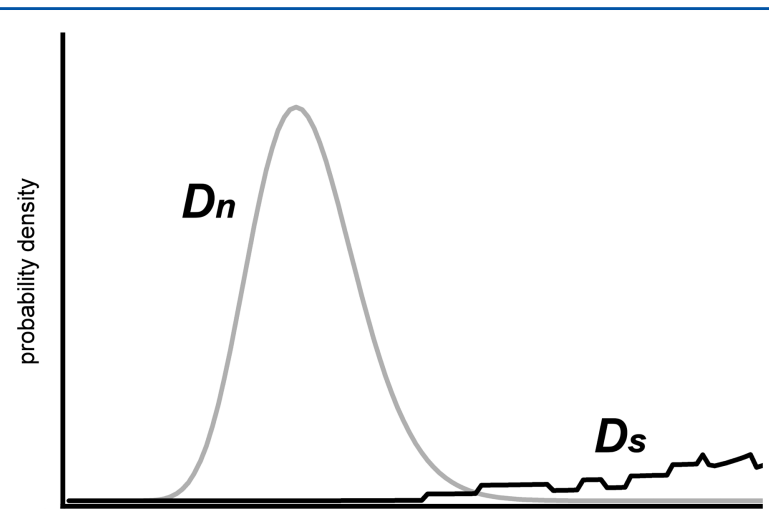

Figure 1. The simulated probability distributions of standard deviations calculated from the spectral points of windows containing noise points only ( $D_{n}$, gray curve) and those containing signal points ( $D_{s}$, black curve).

present in $W_{s}$. The standard deviations calculated from $W_{s} \mathrm{~s}$ are variant and usually much larger than those calculated from $W_{\mathrm{n}} \mathrm{s}$. The two distributions, $D_{\mathrm{n}}$ and $D_{\mathrm{s}}$, are distinct, and their simulations are shown in Figure 1.

Calculating the Expected Value of Noise Standard Deviation $\sigma$. If we model $D_{\mathrm{n}}$ with $S$, we can estimate the expected value of noise standard deviation $\sigma$ from the distribution model. Because the signal standard deviations are usually larger than the noise standard deviations, the mixed distribution $D_{\mathrm{m}}$ will match $D_{\mathrm{n}}$ in the bell-shaped region. We can remove the right tail of $D_{\mathrm{m}}$ and simply use the bell-shaped portion of $D_{\mathrm{m}}$ to simulate $D_{\mathrm{n}}$. BaselineCorrector first calculates a set of standard deviations using a default 41-point sliding window from the full spectrum and identifies a temporary median from this set of standard deviations. The iterative procedures exclude elements that are two times larger than the median, and the new median becomes smaller until it converges. The convergent value is located at the center of the bell-shaped distribution. Because a chi-squared distribution with more than approximately 10 degrees of freedom is close to the normal distribution and is quite symmetric, the median and mean are nearly the same. Thus, the convergent value can be used to represent the expected value of the noise standard deviation $\sigma$. We use the median instead of the mean during the $\sigma$ estimation calculation to avoid the influence of extreme values in the distribution.

Classification of Windows. Once the distribution model of $D_{\mathrm{n}}$ and the expected value $\sigma$ are determined, it is easy to establish a suitable cutoff, expressed as folds of $\sigma$, to classify the sliding windows as $W_{\mathrm{n}}$ or $W_{\mathrm{s}}$. On the basis of the derived chisquared distribution model $S$, we can estimate the percentage of $W_{\mathrm{n}} \mathrm{s}$ via the standard deviations that are lower than the cutoff value. For example, if we use $1.0 \sigma$ (mean of $S$ ) as the threshold, approximately $50 \%$ of $W_{\mathrm{n}}$ will fall below the threshold and, therefore, be included in the baseline calculation. In this study, BaselineCorrector utilized a $1.1 \sigma$ cutoff threshold as the default value for classifying the 41-point sliding windows. Thus, a sliding window with a standard deviation that was equal to or smaller than $1.1 \sigma$ was classified as $W_{\text {n }}$, while a sliding window with a standard deviation greater than $1.1 \sigma$ was classified as $W_{s}$. Theoretically, according to the $S$ model, this distribution (a distribution with a $1.1 \sigma$ cutoff value) should include $83.2 \%$ of the true $W_{\mathrm{n}} \mathrm{s}$.

Classification of Spectral Points. Because the sliding window moves point-by-point, each spectral point, with the exception of the first 40 and the last 40 , may belong to 41 different windows. The 41 different windows that contain a particular spectral point are not necessarily all $W_{\mathrm{n}}$ or all $W_{\mathrm{s}}$. We can assume most of the spectral points in the $W_{\mathrm{n}}$ are noise points. However, it is difficult to further classify the spectral points within a window classified as $W_{s}$ that contains both signal points and noise points. To decrease the ambiguity of spectral point classification and prevent a single isolated point to be classified as signal or noise point, our algorithm defines the central 3-7 points (default: 7 points) of all $W_{s}$ as signal points. If a spectral point is ever defined as a signal point by any $W_{\text {s }}$, the spectral point is classified as a signal point.

Baseline Fitting. After classifying the spectral points, the next procedure is to fit a baseline to the noise points. Because signal intensities will obscure the position of the baseline, it is difficult to estimate the real baseline drifts that occur during the signal segments. However, if the noise points can be identified robustly, a suitable spline function passing through the identified noise points can well estimate the baseline and preserve the signals. BaselineCorrector estimates the baseline in each signal region by interpolating the segment according to the adjacent baseline intensity. Our algorithm replaces the signal regions with linear interpolations to build a signalremoved spectrum and then smooths the signal-removed 
(A)

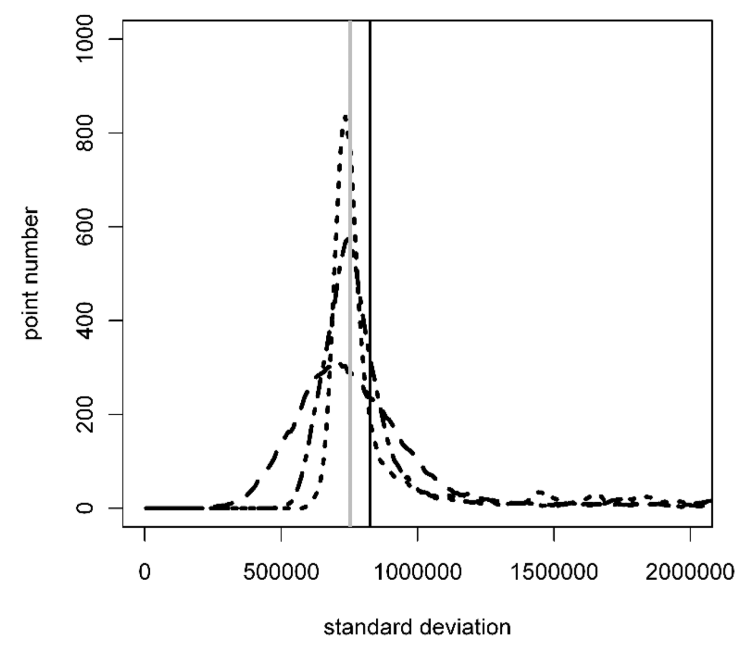

(C)

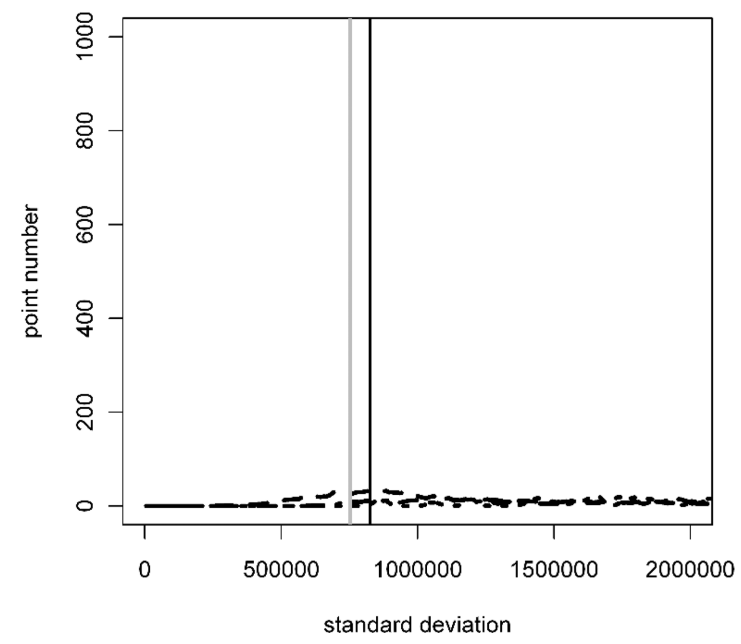

(B)

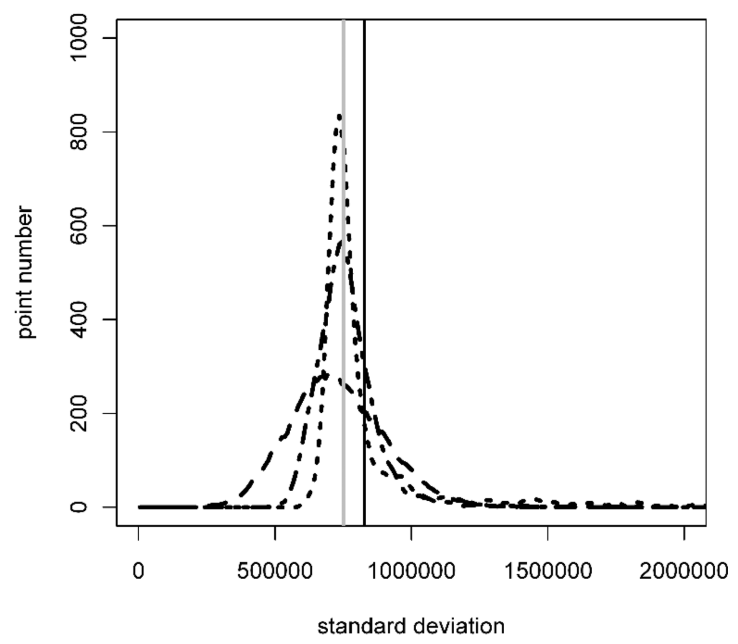

(D)

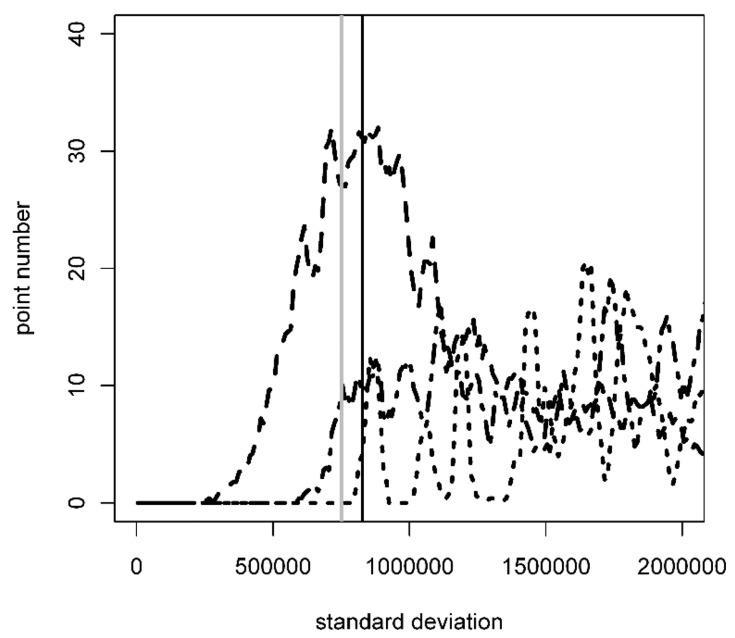

Figure 2. Distributions of $D_{\mathrm{m}}, D_{\mathrm{n}}$, and $D_{\mathrm{s}}$ : (A) $D_{\mathrm{m}} \mathrm{s}$, distributions of standard deviations calculated from all sampled windows (dashed: 11-point window; dot-dashed: 41-point window; dotted: 121-point window); (B) $D_{\mathrm{n}} \mathrm{s}$, distributions of standard deviations calculated from the windows containing noise points only; (C) $D_{s}$ s, distributions of standard deviations calculated from the windows containing signal points; (D) $D_{s} s$ displayed with an amplified scale. The histogram interval was set at 10000 . Standard deviations larger than $2 \times 10^{6}$ were removed. The gray vertical line is the expected value of the noise standard deviation $(\sigma)$ in this spectrum. The black vertical line is the value of $1.1 \sigma$ (the default cutoff value).

spectrum (the default, a 121-point mean filter) to produce the estimated baseline.

\section{MATERIALS AND METHODS}

NMR Analysis of Cell Extracts, Human Plasma, and Urine Samples. Extracts of PC9 human nonsmall cell lung cancer cells and urine samples were prepared for ${ }^{1} \mathrm{H}$ NOESYPR1D NMR spectroscopy analysis. Human plasma samples were prepared for 1D diffusion-edited and CPMG ${ }^{1} \mathrm{H}$ NMR analysis. Details of sample preparation, NMR analysis, and data preprocessing are described in the Supporting Information.

Spiking NMR Experiments. To test the accuracy of BaselineCorrector, we introduced signals of 25 pure compounds into the cell extract spectrum described in the previous section to generate a set of simulated spectra. The 25 pure compounds consist of acetate, alanine, betaine, creatine, creatinine, cytosine, ethanol, formate, fumarate, glutamate, glutamine, glycerol, glycine, hippurate, histidine, leucine, myoinositol, phenol, pyruvate, succinate, taurine, threonine, tyrosine, uracil, and methanol. The signals of spiking compounds distribute over a spectral range of $0.9-8.5 \mathrm{ppm}$.

These pure compound spectra were produced from our laboratory with the same procedures as the cell extract spectrum or downloaded from the Web site of the Human Metabolome Database (HMDB). ${ }^{32,33}$ Each spectrum was manually aligned, phased, baseline-corrected using the software of Chenomx 6.0, and then output into R v. 2.8.1 for further processing. The signal-free segments were set to zero with an intensity threshold. All of the spectra were normalized to an equal maximal intensity. The cell extract spectrum was baselinecorrected with BaselineCorrector, but the signal/noise points were assigned manually with the expert-defined signal/noise points. Ten sets of 25 uniformly distributed random coefficients given with $\mathrm{U}(0.02,0.2)$ were generated. A total of 10 spiked simulated spectra were produced by summation of spectral 
intensities of the cell extract spectrum and the pure compound spectra multiplied by random coefficients. Baseline rolling was reinduced with a set of sine wave functions with angular frequency $2 \pi / 3$ per ppm. The beginnings of sine waves were given randomly, and the oscillation amplitudes were randomly given in a range of one-twentieth to one-thirtieth of the maximal spectral intensity.

The ability of the algorithm to restore the spectral intensities to the original spectral intensities without the sine wave noises was tested. The results of BaselineCorrector were compared with ACD/1D NMR Manager (verson: 10.0, Advanced Chemistry Development, Inc., ACD/Laboratories), MNova (version: 8.0.2, Mestrelab Research SL), and the penalized parametric smoothing model (PSM). ${ }^{20}$ The automatic FID reconstruction function of $1 \mathrm{D}$ NMR Manager and Whittaker smoother function ${ }^{18}$ of MNova were utilized for comparisons. The PSM in this experiment was implemented with an in-house program coded with $\mathrm{R}$. The water noises in 4.5-5.0 ppm were removed for PSM calculation to avoid the influence of negative signals. The relative error of baseline correction in each spiked spectral segment was calculated as follows:

$$
\begin{aligned}
& \text { absolute percentage error }(\%) \\
& =\mid(\text { integral of baseline-corrected simulated spectrum }) \\
& -(\text { integral of cell extract spectrum }) \\
& -(\text { summation of integrals of pure compound spectra }) \mid \\
& \text { / (summation of integrals of pure compound spectra })
\end{aligned}
$$

To compare the effects of different frequency-domain point sizes on the baseline correction efficiency of BaselineCorrector, another set of 25-compound spiked spectra were generated from the same cell extract spectra which were Fouriertransformed from $16 \mathrm{k}, 32 \mathrm{k}$, and zero-filled $64 \mathrm{k}$ time domain data. The corresponding frequency domain point sizes are $0.0006,0.0003$, and $0.00015 \mathrm{ppm}$. The spiking procedures and the details are provided in the Supporting Information. The relative error of baseline correction by BaselineCorrector for each spiked spectral segment with various point sizes was compared.

\section{RESULTS AND DISCUSSION}

Modeling the Standard Deviations Distribution of Noise in BaselineCorrector. The $D_{\mathrm{n}}, D_{\mathrm{s}}$, and $D_{\mathrm{m}}$ determined from a cell extract spectrum are displayed in Figure 2. Similar to the simulated distribution $S$, the $D_{\mathrm{n}} \mathrm{s}$ (Figure 2B) from different window sizes were close to bell-shaped and symmetric. To confirm the fits of $D_{\mathrm{n}}$ and $S$, we overlapped the $D_{\mathrm{n}}$ from a 41point sliding window with the corresponding probability density function of $S$ and found that the two distributions were well matched (Figure S-1, Supporting Information). In contrast, despite the different window size, the $D_{\mathrm{s}} \mathrm{s}$ were flat and widely spread (Figure 2C,D). There was no significant overlap between the $D_{\mathrm{n}} \mathrm{s}$ and the $D_{\mathrm{s}} \mathrm{s}$ from the same window size (Figure 2B,C). Therefore, it was possible to separate the two distributions with a cutoff threshold. In the bell-shaped regions, the mixture distributions, $D_{\mathrm{m}} \mathrm{s}$, were similar to the corresponding $D_{\mathrm{n}} \mathrm{s}$ (Figure 2A,B). The bell-shaped region of $D_{\mathrm{m}} \mathrm{s}$ could be used to model the $D_{\mathrm{n}} \mathrm{s}$ to calculate the expected value of the noise standard deviation $\sigma$. The convergent median value calculated by BaselineCorrector for this spectrum was located close to the center of the bell-shaped area and could be used to represent $\sigma$ (Figure 2A).

Determining of the Sliding Window Size in BaselineCorrector. Choosing a good sliding window size affects the classification of spectral points. BaselineCorrector choose the window size with the statistical distribution model of noise standard deviation. According to the derived chi-squared distribution $S$, the distributions of noise standard deviation from small sliding windows should be relatively diverse, and the distributions from large sliding windows should be more concentrated (Figure S-2, Supporting Information). As predicted, the actual distributions from different sizes of sliding windows are shown in Figure 2B. For a diverse distribution from a small sliding window, a larger cutoff value is required to include the desired ratio of $W_{n}$, and this larger cutoff increases the error and includes more $W_{s}$ s. Therefore, choosing a concentrated distribution from large windows for window classification is essential. However, an oversized sliding window makes $W_{s}$ more likely to contain both signal and noise points and is prone to produce false positives and false negatives in the subsequent classifications of spectral points. On the basis of these results/observations of simulated distributions in Supporting Information Figure S-2, a sliding window size around 40 points is not oversized and its corresponding distribution is concentrated enough to assist the classification of $W_{\mathrm{n}}$ and $W_{\mathrm{s}}$. Therefore, the 41-point window was selected as the default window size.

Estimating of the Expected Value of Noise Standard Deviation in BaselineCorrector. In theory, the intensity values of noise vary randomly and can be defined to be a random variable as a result. The noise standard deviation calculated from random noise is not a constant and can also be regarded as a random variable. However, the expected value $(\sigma)$ of the noise standard deviation is a constant and can be used to represent the noise standard deviation. BaselineCorrector iteratively estimates the expected value of noise standard deviation utilizing the median from the entire distribution. This estimated value was shown to be located at the center of the bell-shaped distribution of the noise standard deviation (Figure 2B) and therefore was a more precise estimation of $\sigma$.

Current baseline correction methods do not estimate $\sigma$ with the true noise standard deviation distribution. Golotvin's method breaks the spectrum into 32 segments and uses the minimum value of the standard deviations of these segments as an estimate of $\sigma .^{19}$ Although this estimate may approximate $\sigma$, this method is hampered by several factors. If peaks are distributed within all 32 regions, this method will overestimate $\sigma$. In contrast, if there are few or no peaks in some of the 32 regions, the method will underestimate $\sigma$. On the other hand, Xi's method assumes that the noise variance is approximately equal to the variance of the segments with small mean values. ${ }^{20}$ Thus, the spectrum is divided into several small regions, and the LOWESS regression is used to fit the means and variances of these regions using the predicted value of the variance at zero mean as an estimate of the noise variance. The Xi's method usually provides a good estimate of $\sigma$ in spectra having baselines near zero. However, with markedly downward shifted spectra, some signal variances will have mean values near zero, causing this method to overestimate $\sigma$. Furthermore, when the baseline is markedly shifted upward, signal-free regions will have mean values far from zero and prone to underestimate $\sigma$.

Because the metabolomic NMR spectra are complicated with mixed signal and noise segments, to estimate $\sigma$ correctly is 
somewhat difficult. However, the accuracy of the $\sigma$ estimation significantly affects the quality of the baseline calculation. Therefore, prediction of $\sigma$ should be as precise as possible. BaselineCorrector estimates $\sigma$ by sampling all standard deviations in each segment window of the entire spectrum to obtain their distribution. Thus, this estimation, based on the entire distribution, is not affected by sampling biases or shifted spectral baselines.

BaselineCorrector uses a threshold for all standard deviations to classify the spectral points, while other studies utilize the intensity difference or the first derivative as the judgment criteria to classify the spectral points. ${ }^{17,19}$ Most classification algorithms using intensity difference are straightforward and rapidly calculated. The concept behind these is the intensity difference will be larger in the presence of a signal. From the earlier discussion, we demonstrated that having a distribution model can help determine suitable classification parameters that minimize classification errors. Since the intensity difference calculated from a given window is still a random variable, using a distribution to model this variable and facilitate the classification is feasible. However, there are several disadvantages to model the distributions of intensity difference of noise. One of the disadvantages is there is no convenient statistical model to fit these distributions. The second disadvantage is, unlike the constant expected values of noise standard deviation from different window sizes, the expected values of the intensity difference of noise from different window sizes are not constant. The expected values of the intensity difference of noise increase as the window size increases. Because of these disadvantages, it is difficult to determine suitable parameter settings (including window size and cutoff threshold) using distribution models of noise intensity difference. Therefore, we utilized noise standard deviation instead of intensity difference as the criteria to classify spectral points.

Determining a Suitable Cutoff Value to Classify Each Sliding Window in BaselineCorrector. BaselineCorrector uses $1.1 \sigma$ as the default best threshold to classify sliding windows. However, misclassification of $W_{\mathrm{s}} \mathrm{s}$ as $W_{\mathrm{n}} \mathrm{s}$ occurs when the $W_{\mathrm{s}} \mathrm{s}$ standard deviations fall below the cutoff threshold. Classifying true signal points as noise points will make the estimated baseline pass through the signal regions of a spectrum and offset the signal during the process of baseline correction. Therefore, the cutoff threshold should be as low as possible to avoid this type of misclassification. However, the cutoff threshold should also be large enough to include adequate noise points for baseline building. From our experience in metabolomics studies, a cutoff including around $80 \%$ to $90 \%$ of $W_{\mathrm{n}}$ is required for good baseline building. According to the statistical model of $D_{\mathrm{n}}$, a cutoff around $1.1 \sigma$ is the best cutoff threshold which minimizes the misclassification and simultaneously includes adequate noise points. Consistent with our model, Figure 3 shows the sensitivity to efficiently include most of the noise points ranges from 1.1 to $1.2 \sigma$ cutoff in this example spectrum. Figure 3 also shows the specificity to classify the true signal points as signal points gradually decreases when the cutoff threshold increases and exceeds 1.0 $\sigma$. Therefore, to minimize the error, the default $1.1 \sigma$ cutoff is the best of choice. At the default $1.1 \sigma$ cutoff, the misclassification rate was $0.4 \%$ in Figure 3 when compared with an expert's classification. With the cutoff thresholds including $85 \%$ of true noise points, the window size around 40 points has the lowest error rate to misclassify the true signal points with the example cell extract spectrum, therefore,

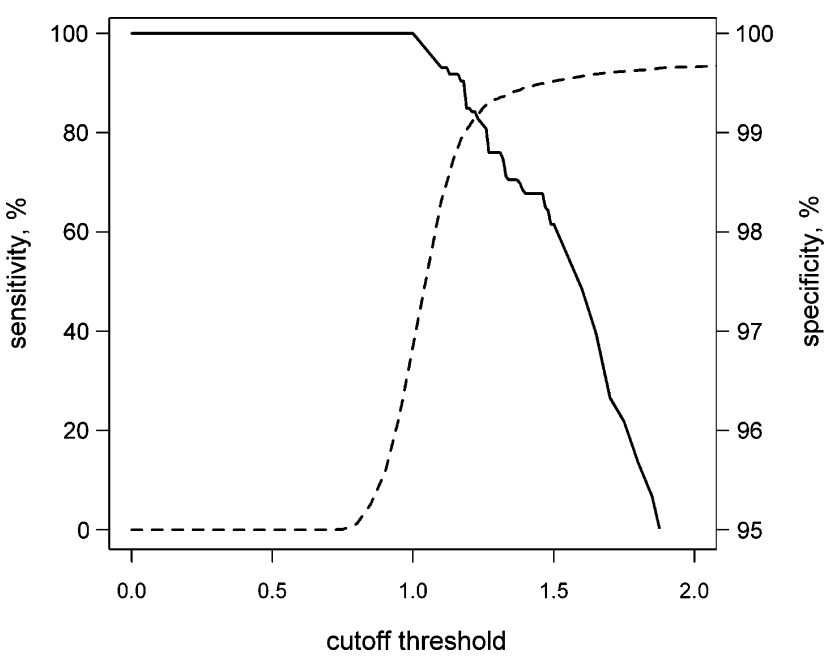

Figure 3. The sensitivity and specificity of spectral point classification at different cutoff thresholds. The sensitivity (dashed curve) is calculated as (number of true noise point classified as noise point)/ (number of true noise point). The specificity (solid curve) is calculated as (number of true signal point classified as signal point)/(number of true signal point). The cutoff threshold is expressed as a multiplicative of the noise standard deviation $(\times \sigma)$ ).

considered the optimal window size (Figure S-3, Supporting Information). It is consistent with the theoretical estimation described in previous sections.

BaselineCorrector Can Preserve the Low Intensity Signals with the Cutoff Derived from the Statistical Model. To test the signal detection ability of our algorithm, we created a model with a set of 41-point segments containing artificial, random, normal noise, and a superimposed peak, simulated by a Lorentzian function, in the center of the window. The sampling rate was 1500 spectral points per ppm, and the full width at half-maximum of the low-intensity peak occurred at $0.01 \mathrm{ppm}$, which is equal to the length of 15 spectral points in the simulated segment. Using peak intensities from 0 to $5 \sigma$, the standard deviations of the peak-containing segments were calculated in the presence of 1000 simulated random noise segments. The results indicated that our algorithm can detect approximately $60 \%$ of the peaks with heights of $2 \sigma$, more than $90 \%$ of the low intensity peaks with heights of $3 \sigma$ and nearly $100 \%$ of the peaks with heights of $4 \sigma$ (Figure S-4, Supporting Information). Because our algorithm uses a wide mean filter to smooth the signal-removed spectra, the calculated baselines are not significantly affected by small, undetected peaks. Therefore, the low-intensity peaks will not be offset by BaselineCorrector during the baseline correction processes.

Distribution Model of Noise Standard Deviation and the Derived Classification Parameters in BaselineCorrector Can Be Universally Used in a Variety of 1D NMR Spectra. BaselineCorrector models the distribution of noise standard deviation to determine the parameters for signal/noise classification. The robustness of BaselineCorrector depends on the universal property of the applied distribution model. In contrast to spectral signal intensities, which vary greatly in different spectra and cannot be predicted or modeled, the distributions of noise intensities consistently follow a Gaussian distribution $^{34}$ even in different NMR experiments. The advantage of our approach is to ignore the unpredictable signal portion of the spectra and model the noise part only. That is, 
(A)

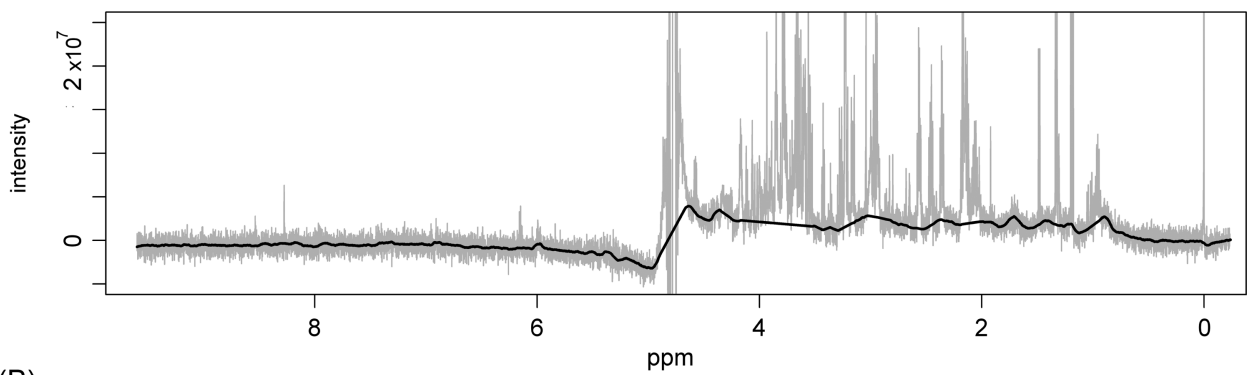

(B)

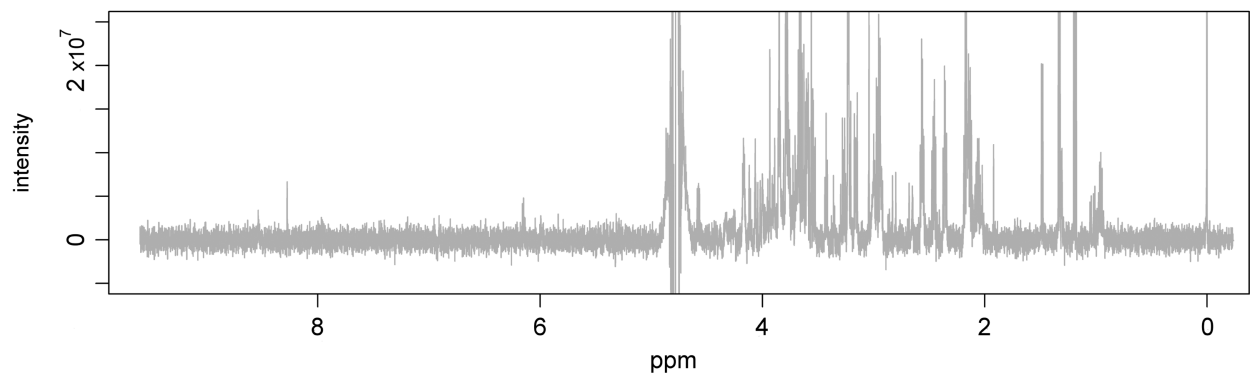

Figure 4. (A) A ID NOESY NMR spectrum of cell extracts and the baseline estimated by BaselineCorrector. (B) Spectrum of (A) after baseline correction.

regardless of parameters such as machine frequency, acquisition time, sampling rate, point numbers, apodization, zerofilling, etc applied in NMR experiments, the noise intensities will have the same distribution with potentially different mean values. The standard deviations of true noise intensities in different spectra, when normalized to the same mean value, will follow the same model of distributions. Therefore, on the basis of a stable model, BaselineCorrector is able to maintain its performance in a variety of situations.

Baseline Correction Results of a 1D NOESY NMR Spectrum. The 1D NOESY NMR spectrum of a human cancer cell extract before baseline correction is shown in Figure 4A. This spectrum exhibits crowded signals interrupted by signal-free segments. The water noise was not removed. The baseline drift was larger than many of the signal intensities. The black line shows the baseline estimated by BaselineCorrector. The estimated baseline is smooth and fits the signal-free regions well. Although it was left uncorrected by our algorithm, the substantial water noise did not affect the baseline estimations of neighboring regions. Because this solvent segment is usually discarded during subsequent data management, it is not necessary to remove the solvent noise before the baseline correction process. The tiny signals were preserved, and the crowded broad peaks were not flattened by the baseline correction process. The baseline-corrected spectrum is shown in Figure 4B. Using a personal computer (Windows XP operation system, Intel Core2 Duo E8400 CPU, and 2GB memory), the calculation time for this 19200-point length spectrum is around $1.8 \mathrm{~s}$ using BaselineCorrector coded in R.

Using the same spectrum, we showed the baselines from BaselineCorrector and the method implemented by Chenomx 6.0 (Figure 5A,B, respectively). In Figure 5A,B, BaselineCorrector capably detects most of the signal-free segments and makes the estimated baseline to pass through the center of baseline noise.

Baseline Correction Results of a Diffusion-Edited NMR Spectrum. A diffusion-edited spectrum of a human plasma sample and the associated baselines estimated by Base-
(A)

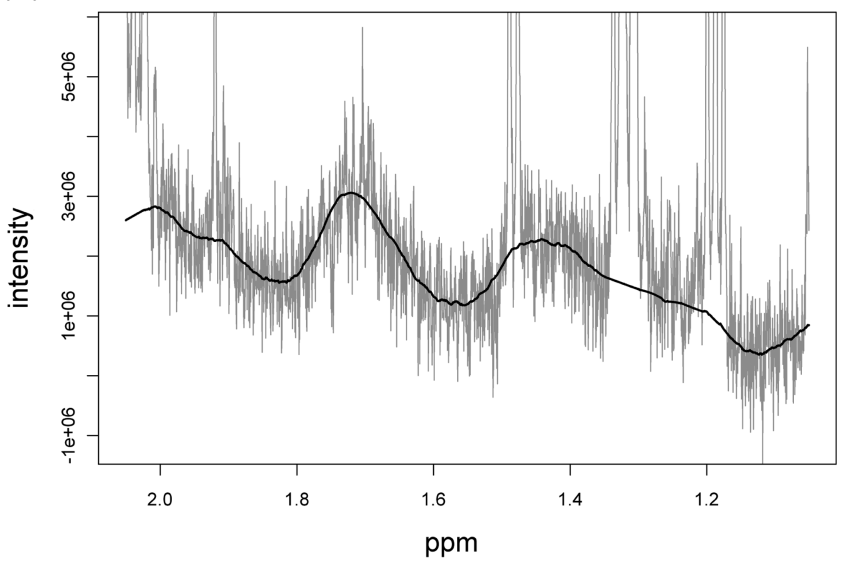

(B)

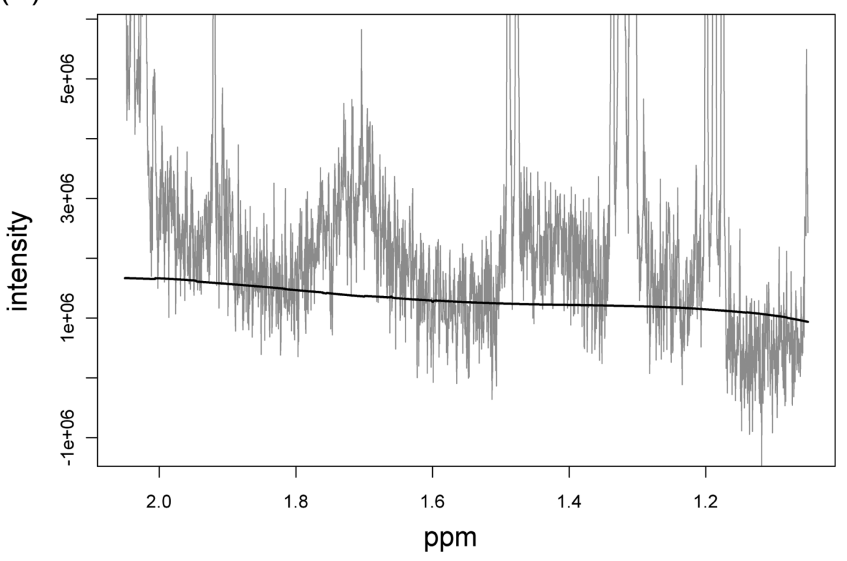

Figure 5. Comparison of two baseline estimation methods: (A) BaselineCorrector and (B) Chenomx 6.0 using an automatic spline function without manual correction.

lineCorrector are shown in detail in the Supporting Information (Figure S-5). 
Baseline Correction Results of Spiking Experiments. A spiked simulated spectra and its baseline determined by BaselineCorrector are shown in Figure 6. The determined

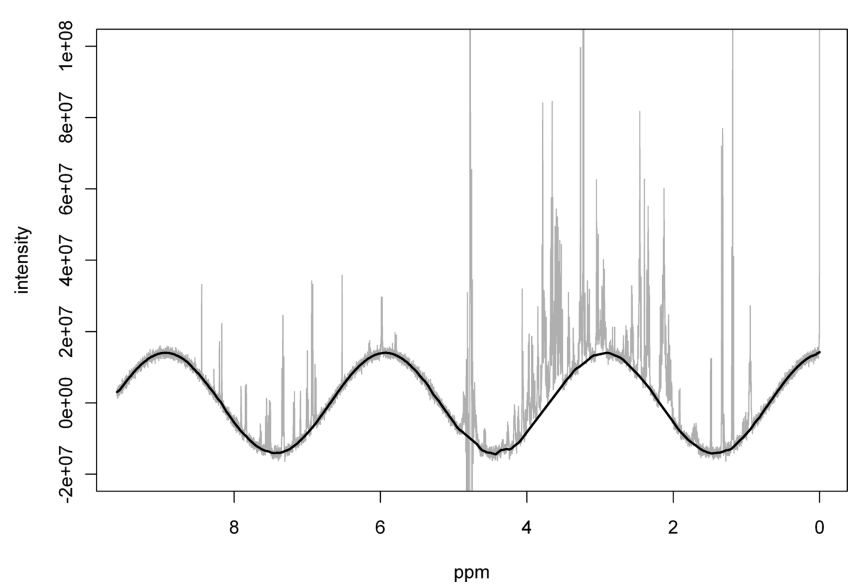

Figure 6. An example of a spiked simulated spectrum and its baseline estimated by BaselineCorrector. The baseline rolling was produced artificially with a sine wave function.

baseline well matches the sine wave oscillation in most of spectral segments. Among MNova, PSM, ACD/1D NMR Manager, and BaselineCorrector, BaselineCorrector has the lowest mean absolute percentage error of $5.2 \%$ calculated from all spiked spectral segments (Table S-1, Supporting Information). The mean absolute percentage error of ACD/1D NMR Manager is $10.5 \%$; MNova, $19.4 \%$; and PSM, 24.9\%. One example of baselines determined by the four algorithms is shown in the Supporting Information (Figure S-6). Compared with other three algorithms, the baseline estimated by BaselineCorrector better follows the original baseline and less attenuates the broad and overlapping peaks. The detailed absolute percentage errors calculated from each spiked spectral segment are shown in the Supporting Information (Table S-1).

To select the optimal values of window size and cutoff threshold, the means of relative errors calculated from different combinations of the window size and cutoff threshold are shown with a heat map in Figure 7 (in grayscale) and in Supporting Information Figure S-7 (in color). Consistent with our default values, the combination of 41-point window and 1.1 $\sigma$ cutoff has the lowest error value. From this simulated example, the optimal value of window size ranged from 30 to 40 points, and the cutoff ranged from 1.1 to $1.2 \sigma$.

Another example of the spiked simulated spectra which were Fourier-transformed from 16k, 32k, and zero-filled 64k time domain data and their baselines determined by BaselineCorrector are shown in Supporting Information Figure S-8. The determined baselines are similar to each other for the three spectra with different spectral density. The mean absolute percentage errors of baseline correction by BaselineCorrector for $16 \mathrm{k}, 32 \mathrm{k}$, and $64 \mathrm{k}$ spectra are $3.3 \%, 2.5 \%$, and $3.5 \%$, respectively. The results show BaselineCorrector is able to maintain its baseline correction efficiency in the spectra at various frequency domain point sizes. Details of baseline correction data for all integrated spiked segments are shown in Supporting Information Table S-2.

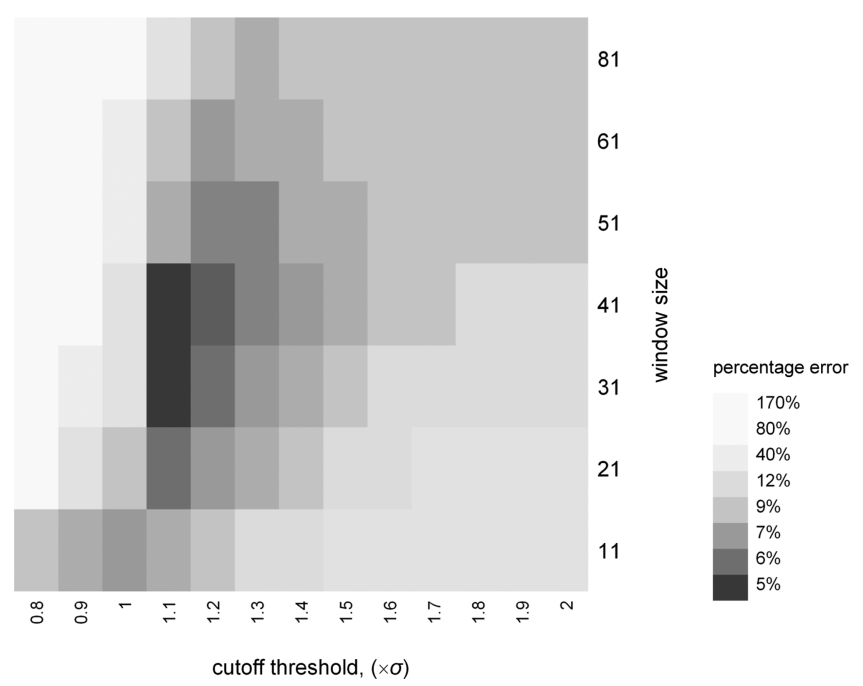

Figure 7. Heat map (in grayscale) of mean absolute percentage errors of baseline correction results using different combinations of window sizes and cutoff thresholds.

\section{CONCLUSIONS}

With the implementations described above, we have demonstrated that BaselineCorrector can successfully estimate the baselines of complicated $1 \mathrm{D}{ }^{1} \mathrm{H}$ NMR metabolomic spectra and diffusion-edited NMR metabolomic spectra. The most significant contribution of this novel algorithm is its use of a statistical distribution model to generate the least-error in signal/noise classification. According to this model, the BaselineCorrector-optimized classification parameters may be universally applied to various types of $1 \mathrm{D}$ NMR experiments. User readjustment of parameters for each spectrum is usually not required; although, in some cases, mild adjustments of cutoff threshold and window size around the optimal defaults may be beneficial for special protocols or machines. By minimizing baseline errors, BaselineCorrector is able to preserve low signal peaks and correctly handle wide, overlapping peaks in complex metabolomic spectra.

\section{ASSOCIATED CONTENT}

S Supporting Information

Additional information as noted in text. This material is available free of charge via the Internet at http://pubs.acs.org.

\section{AUTHOR INFORMATION}

\section{Corresponding Author}

*Phone: +886-2-33664888 ext.529. Fax: +886-2-33663754. Email: yjtseng@csie.ntu.edu.tw.

\section{Notes}

The authors declare no competing financial interest.

\section{ACKNOWLEDGMENTS}

The authors thank reviewers for the comments and suggestions. This work was funded by the Taiwan National Science Council, grant numbers 100-2325-B-002-004- and 101-2325-B-002-005-, and project SKH-8302-93-3103 from Shin-Kong Wu Ho-Su Memorial Hospital. Resources of the Laboratory of Computational Molecular Design and Metabolomics and the Department of Computer Science and Information Engineering of National Taiwan University were used to perform these studies. 


\section{REFERENCES}

(1) Lindon, J. C.; Holmes, E.; Nicholson, J. K. Expert. Rev. Mol. Diagn. 2004, 4, 189-199.

(2) Beckonert, O.; Keun, H. C.; Ebbels, T. M.; Bundy, J.; Holmes, E.; Lindon, J. C.; Nicholson, J. K. Nat. Protoc. 2007, 2, 2692-2703.

(3) Zhang, S.; Nagana Gowda, G. A.; Asiago, V.; Shanaiah, N.; Barbas, C.; Raftery, D. Anal. Biochem. 2008, 383, 76-84.

(4) Yang, Y.; Li, C.; Nie, X.; Feng, X.; Chen, W.; Yue, Y.; Tang, H.; Deng, F. J. Proteome Res. 2007, 6, 2605-2614.

(5) Weljie, A. M.; Newton, J.; Mercier, P.; Carlson, E.; Slupsky, C. M. Anal. Chem. 2006, 78, 4430-4442.

(6) Nicholson, J. K.; Lindon, J. C.; Holmes, E. Xenobiotica 1999, 29, 1181-1189.

(7) Nicholson, J. K.; Wilson, I. D. Nat. Rev. Drug Discovery 2003, 2, 668-676.

(8) Clayton, T. A.; Lindon, J. C.; Cloarec, O.; Antti, H.; Charuel, C.; Hanton, G.; Provost, J. P.; Le Net, J. L.; Baker, D.; Walley, R. J.; Everett, J. R.; Nicholson, J. K. Nature 2006, 440, 1073-1077.

(9) Claudino, W. M.; Quattrone, A.; Biganzoli, L.; Pestrin, M.; Bertini, I.; Di Leo, A. J. Clin. Oncol. 2007, 25, 2840-2846.

(10) Lewis, G. D.; Asnani, A.; Gerszten, R. E. J. Am. Coll. Cardiol. 2008, 52, 117-123.

(11) Hughesa, D. G.; Lindblomb, G. J. Magn. Reson. 1977, 26, 469479.

(12) Zau, G.; Torchia, D. A.; Bax, A. J. Magn. Reson. Ser. A 1993, 105, 219-222.

(13) Marion, D.; Bax, A. J. Magn. Reson. 1988, 79, 352-356.

(14) Heuer, A.; Haeberlen, U. J. Magn. Reson. 1989, 85, 79-84.

(15) Tang, C. J. Magn. Reson. 1994, 109, 232-240.

(16) Pearson, G. A. J. Magn. Reson. 1977, 27, 265-272.

(17) Dietrich, W.; Rudel, C. H.; Neumann, M. J. Magn. Reson. 1991, 91, 1-11.

(18) Cobas, J. C.; Bernstein, M. A.; Martin-Pastor, M.; Tahoces, P. G. J. Magn. Reson. 2006, 183, 145-151.

(19) Golotvin, S.; Williams, A. J. Magn. Reson. 2000, 146, 122-125.

(20) Xi, Y.; Rocke, D. M. BMC Bioinf. 2008, 9, 324.

(21) Chang, D.; Banack, C. D.; Shah, S. L. J. Magn. Reson. 2007, 187, 288-292.

(22) Bartels, C.; Güntert, P.; Wüthrich, K. J. Magn. Reson. Ser. A 1995, 117, 330-333.

(23) Guntert, P.; Wuthrich, K. J. Magn. Reson. 1992, 96, 403-407.

(24) Brown, D. E. J. Magn. Reson. Ser. A 1995, 114, 268-270.

(25) Wang, T.; Shao, K.; Chu, Q.; Ren, Y.; Mu, Y.; Qu, L.; He, J.; Jin, C.; Xia, B. BMC Bioinf. 2009, 10, 83.

(26) Izquierdo-Garcia, J. L.; Rodriguez, I.; Kyriazis, A.; Villa, P.; Barreiro, P.; Desco, M.; Ruiz-Cabello, J. BMC Bioinf. 2009, 10, 363.

(27) Zhang, Z. M.; Chen, S.; Liang, Y. Z. Analyst 2010, 135, 11381146.

(28) Bao, Q.; Feng, J.; Chen, F.; Mao, W.; Liu, Z.; Liu, K.; Liu, C. J. Magn. Reson. 2012, 218, 35-43.

(29) http://www.inmr.net.

(30) Liu, M.; Nicholson, J. K.; Lindon, J. C. Anal. Chem. 1996, 68, $3370-3376$

(31) Rocke, D. M.; Lorenzato, S. Technometrics 1995, 37, 176-184. (32) Wishart, D. S.; Tzur, D.; Knox, C.; Eisner, R.; Guo, A. C.; Young, N.; Cheng, D.; Jewell, K.; Arndt, D.; Sawhney, S.; Fung, C.; Nikolai, L.; Lewis, M.; Coutouly, M. A.; Forsythe, I.; Tang, P.; Shrivastava, S.; Jeroncic, K.; Stothard, P.; Amegbey, G.; Block, D.; Hau, D. D.; Wagner, J.; Miniaci, J.; Clements, M.; Gebremedhin, M.; Guo, N.; Zhang, Y.; Duggan, G. E.; Macinnis, G. D.; Weljie, A. M.; Dowlatabadi, R.; Bamforth, F.; Clive, D.; Greiner, R.; Li, L.; Marrie, T.; Sykes, B. D.; Vogel, H. J.; Querengesser, L. Nucleic Acids Res. 2007, 35, D521-526.

(33) Wishart, D. S.; Knox, C.; Guo, A. C.; Eisner, R.; Young, N.; Gautam, B.; Hau, D. D.; Psychogios, N.; Dong, E.; Bouatra, S.; Mandal, R.; Sinelnikov, I.; Xia, J.; Jia, L.; Cruz, J. A.; Lim, E.; Sobsey, C. A.; Shrivastava, S.; Huang, P.; Liu, P.; Fang, L.; Peng, J.; Fradette, R.; Cheng, D.; Tzur, D.; Clements, M.; Lewis, A.; De Souza, A.; Zuniga, A.; Dawe, M.; Xiong, Y.; Clive, D.; Greiner, R.; Nazyrova, A.;
Shaykhutdinov, R.; Li, L.; Vogel, H. J.; Forsythe, I. Nucleic Acids Res. 2009, 37, D603-610.

(34) Adams, M. J. Chemometrics in Analytical Spectroscopy, 2nd ed.; Royal Society of Chemistry: Cambridge, U.K., 2004; p 34. 